\title{
A NOTE ON PSEUDOCONVEXITY AND PROPER HOLOMORPHIC MAPPINGS
}

\author{
E. B. LIN AND B. WONG
}

(Communicated by Irwin Kra)

\begin{abstract}
In this paper we discuss some connections between proper holomorphic mappings between domains in $\mathbb{C}^{n}$ and the boundary behaviors of certain canonical invariant metrics. A compactness theorem has been proved. This generalizes slightly an earlier result proved by the second author.
\end{abstract}

Introduction. A continuous mapping $f: X_{1} \rightarrow X_{2}$ between two topological spaces is called proper if $f^{-1}(K) \subset X_{1}$ is compact whenever $K \subset X_{2}$ is compact. Proper holomorphic mappings between analytic spaces stand out for their beauty and simplicity. For instance, if $g: D_{1} \rightarrow D_{2}$ is a proper holomorphic mapping between two bounded domains in $\mathbb{C}^{n}$, a theorem of Remmert says that $\left(D_{1}, g, D_{2}\right)$ is a finite branching cover. The branching locus in $D_{1}$ is described by $\left\{z \in D_{1} \mid\right.$ $\operatorname{det}(d g(z))=0\}$. For the past ten years, there has been a great amount of activity in characterizing the proper holomorphic mappings between pseudoconvex domains. It has been known for a long time that there are numerous proper holomorphic maps between unit disks in $\mathbb{C}^{1}$. The simplest example is $g: \Delta=\left\{z \in \mathbb{C}^{1}|| z \mid<1\right\} \rightarrow \Delta$, $g(z)=z^{n}$, where $n$ is any positive integer. Nevertheless, such a phenomenon is no longer true in higher-dimensional cases. H. Alexander was able to verify the following interesting fact.

THEOREM $1[\mathbf{1}]$. Let $B_{n}=\left\{\left.\left(z_{1}, z_{2}, \ldots, z_{n}\right)\left|\sum_{i=1}^{n}\right| z_{i}\right|^{2}<1\right\}$ be the unit ball in $\mathbb{C}^{n}, n \geq 2$. Suppose $f: B_{n} \rightarrow B_{n}$ is a proper holomorphic mapping. Then $f$ must be a biholomorphism.

The following result due to S. Pincuk is an extension of Alexander's theorem.

THEOREM $2[5]$. Let $D_{1}$ and $D_{2}$ be two strongly pseudoconvex bounded domains with smooth boundaries in $\mathbb{C}^{n}, n \geq 2$. Suppose $f: D_{1} \rightarrow D_{2}$ is a proper holomorphic mapping. Then $f$ is a covering.

In [7] the second author proved the following result concerning biholomorphic groups of strongly pseudoconvex domains.

THEOREM 3 [7]. Let $D$ be a strongly pseudoconvex bounded domain with smooth boundary in $\mathbb{C}^{n}$. Then $\operatorname{Aut}(D)$ is noncompact iff $D$ is biholomorphic to $B_{n}, n=$ $\operatorname{dim}_{\mathrm{C}} D$.

Received by the editors June 3, 1986 and, in revised form, December 30, 1986.

1980 Mathematics Subject Classification (1985 Revision). Primary 53C55; Secondary 32A17.

Key words and phrases. Proper holomorphic mappings, strong pseudoconvexity, intrinsic measures. 
In view of a lot of recent attention on the topic of proper holomorphic mappings, the authors feel that it might be worthwhile to point out the following startling fact which generalizes Theorem 3 .

THEOREM 4. Let $D_{1}$ and $D_{2}$ be two strongly pseudoconvex bounded domains with smooth boundaries in $\mathbb{C}^{n}, n \geq 2$. Then $P\left(D_{1}, D_{2}\right)$ is noncompact iff both $D_{1}$ and $D_{2}$ are biholomorphic to $B_{n}$, where $P\left(D_{1}, D_{2}\right)$ denotes the set of all proper holomorphic mappings between $D_{1}$ and $D_{2}$.

Pincuk's Theorem 2 says that proper holomorphic mappings between strongly pseudoconvex domains are unbranching. It follows that Theorem 4 is an immediate consequence of the local version stated next, which is the principal result of this note.

THEOREM 5. Let $D_{1}$ and $D_{2}$ be bounded domains in $\mathbb{C}^{n}$. We denote $P_{0}\left(D_{1}, D_{2}\right)$ as the set of all unbranching proper holomorphic maps from $D_{1}$ to $D_{2}$. Suppose the following two conditions are fulfilled.

(1) There is a strongly pseudoconvex boundary point $p \in \partial D_{2}$.

(2) There exists a point $x \in D_{1}$ and a sequence $\left\{f_{j}\right\} \subseteq P_{0}\left(D_{1}, D_{2}\right)$ such that $\left\{f_{j}(x)\right\}$ converges to $p$.

Then both $D_{1}$ and $D_{2}$ are biholomorphic to $B_{n}$.

(A) Some preliminaries and related results. Let $M$ be a complex manifold of dimension $n, x \in M$, and $k$ an integer between one and $n$.

DEFINITION. The Eisenman differential $k$-measure on $M$ is a function $E_{M}^{k}$ :

$\bigwedge^{k} T(M) \rightarrow \mathbf{R}$ such that for all $(x, v) \in \bigwedge^{k} T_{x}(M)$, $E_{M}^{k}(x, v)=\inf \left\{R^{-2^{k}} \mid\right.$ there exists a holomorphic map $f: B_{k}(R) \rightarrow M$ such that $f(0)=x$ and $\left.d f_{0}\left(\frac{\partial}{\partial w_{1}} \wedge \frac{\partial}{\partial w_{2}} \wedge \cdots \wedge \frac{\partial}{\partial w_{k}}(0)\right)=v\right\}$, where $B_{k}(R)=\left\{w=\left.\left(w_{1}, w_{2}, \ldots, w_{k}\right) \in C^{k}\left|\sum_{i=1}^{k}\right| w_{i}\right|^{2}<R\right\}$.

When $k=1$, it is called a Kobayashi-Royden differential metric [6], denoted $K_{M}=k \sqrt{E_{M}^{1}}$. As $k=n$, it is a volume form, denoted by $E_{M}^{n}=\left|E_{M}^{n}\right| d z_{1} \wedge d \bar{z}_{1} \wedge$ $\cdots \wedge d z_{n} \wedge d \bar{z}_{n}$, where $\left|E_{M}^{n}\right|$ is a function on $M$.

On the other hand, the Carathéodory differential $k$-measure $C_{M}^{k}$ is defined as follows.

DEFinition. $C_{M}^{k}: \bigwedge^{k} T_{x}(M) \rightarrow \mathbf{R},(x, v) \in \bigwedge_{k} T_{x}(M), C_{M}^{k}(x, v)=\sup \left\{1 / R^{2^{k}}\right.$ there exists a holomorphic mapping $f: M \rightarrow B_{k}(R)$ such that $f(x)=0, d f_{x}(v)=$ $\left.\partial / \partial w_{1} \wedge \cdots \wedge \partial / \partial w_{k}(0)\right\}$.

When $k=1$, it is called a Carathéodory-Reiffen differential metric, denoted by $C_{M}=\sqrt{C_{M}^{1}}$. As $k=n$, it is a volume form $C_{M}^{n}=\left|C_{M}^{n}\right| d z_{1} \wedge d \bar{z}_{1} \wedge \cdots \wedge d z_{n} \wedge d \bar{z}_{n}$, where $\left|C_{M}^{n}\right|$ is a function on $M$.

One can also define $E_{M}^{k}$ and $C_{M}^{k}$ relative to a polydisc instead of a ball. They are different measures, but enjoy similar properties. In the sequel, we shall use $I_{M}^{k}$ to represent either $E_{M}^{k}$ or $C_{M}^{k}$.

The following theorem follows almost immediately from the definitions [4]. 
THEOREM (a). (1) $E_{M}^{k} \geq C_{M}^{k}$ on any complex manifold $M$.

(2) Let $f: M_{1} \rightarrow M_{2}$ be a holomorphic mapping between complex manifolds $M_{1}$ and $M_{2}$. Then one has $I_{M_{1}}^{k} \geq f^{*}\left(I_{M_{2}}^{k}\right)$, a measure-decreasing property under $f$.

(3) Let $X$ be a domain of a complex manifold $Y$. Then $I_{X}^{k} \geq I_{Y}^{k}$, a monotone property, holds.

(4) Any biholomorphism $f$ of a complex manifold $X$ is measure-preserving relative to $I_{X}^{k}$, that is, $I_{X}^{k}=f^{*}\left(I_{X}^{k}\right)$.

(5) Let $\widetilde{M}$ be a covering of a complex manifold $M$. Denote $\pi: \widetilde{M} \rightarrow M$ as the covering projection. Then $E_{\widetilde{M}}^{k}=\pi^{*}\left(E_{M}^{k}\right)$.

THEOREM (b) $[3,8]$. Let $D$ be a bounded domain in $\mathbb{C}^{n}$ with a strongly pseudoconvex boundary point $p \in \partial D$. We denote $\widetilde{D}=V \cap D$, where $p \in V$ is a sufficiently small ball in $\mathbb{C}^{n}$. Then the following is true: $\left|E_{\widetilde{D}}^{n}(z)\right| /\left|C_{\widetilde{D}}^{n}(z)\right|$ approaches one as $z \rightarrow p$.

In [7], the next theorem was proved for the special case where $D$ is completely hyperbolic. Actually, a similar proof can yield a slightly more general statement as follows.

THEOREM (c) [7]. Let $D$ be a bounded domain in $\mathbb{C}^{n}$. Suppose that there is one point $x \in D$ such that $\left|E_{D}^{n}(x)\right|=\left|C_{D}^{n}(x)\right|$. Then $D$ is biholomorphic to the euclidean ball.

THEOREM (d) [2] (CARTAN'S FIXED POINT THEOREM). Let $\left(X, d s^{2}\right)$ be a simply-connected complete Riemannian manifold with nonpositive sectional curvature. Suppose $G$ is a compact Lie group acting on $X$ as isometries. Then $G$ has a fixed point.

In particular, any finite group $H$ acting on $X$ isometrically must fix at least one point.

THEOREM (e). Let $D_{1}$ and $D_{2}$ be bounded domains in $\mathbb{C}^{n}$. Suppose that

(1) there is a strongly pseudoconvex point $p \in \partial D_{2}$;

(2) one can find $x \in D_{1}$ and a sequence of holomorphic mappings $\left\{f_{j}\right\} \subset$ $\operatorname{Hol}\left(D_{1}, D_{2}\right)$ such that $\left\{f_{j}(x)\right\} \rightarrow p$.

Then there exists a subsequence of $\left\{f_{j}\right\}$, denoted by the same notation $\left\{f_{j}\right\}$, satisfying the property: For any compact set $K \subset D_{1}$ and any open set $\widetilde{D}=V \cap D_{2}$, where $p \in V$ is an open set in $\mathbb{C}^{n}$, there is a $j_{0}$ in such a way that $f_{j}(K) \subset \widetilde{D}$ for all $j \geq j_{0}$.

PROOF. Since $\left\{f_{j}(x)\right\} \rightarrow p$, by normal family argument one can find a subsequence of $\left\{f_{j}\right\}$ converging on compacta to a holomorphic mapping $f: D_{1} \rightarrow \mathbb{C}^{n}$ so that $f(x)=p$ and $f\left(D_{1}\right) \subseteq \partial D_{2}$. By assumption, $\partial D_{2}$ is strongly pseudoconvex at $p$ and it contains no complex analytic variety of positive dimension through $p$. This implies $f$ is a constant mapping which brings the whole $D$ onto a single point. Our claim in Theorem (e) should now be clear.

(B) Proof. Let us assume $\left|E_{D_{1}}^{n}(x)\right|=\left|C_{D_{1}}^{n}(x)\right|$ for the given point $x$ in $D_{1}$. By Theorem (c), this implies that $D_{1}$ must be biholomorphic to $B_{n}$. If the order of the covering $f_{j}: B_{n}=D_{1} \rightarrow D_{2}$ is greater than one, this would contradict Cartan's fixed point theorem (Theorem (c)) because the Bergman metric on $B_{n}$ has 
negative sectional curvature and it is invariant under biholomorphisms. Thus $D_{2}$ is also biholomorphic to $B_{n}$. Therefore, the whole proof depends on the following assertion.

Claim. $\left|E_{D_{1}}^{n}(x)\right|=\left|C_{D_{1}}^{n}(x)\right|$.

PROOF. For each $j, f_{j}: D_{1} \rightarrow D_{2}$ is a covering. From Theorem (a)(5) we have

$$
E_{D_{1}}^{n}(x, v)=E_{D_{2}}^{n}\left(x_{j}, d f_{j}(v)\right)
$$

where $x_{j}=f_{j}(x)$ and $(x, v)$ is a nonzero $n$-vector at $x$. Let $\left(D_{1}\right)_{k}$ be an increasing sequence of domains such that $\bigcup_{k=1}^{\infty}\left(D_{1}\right)_{k}=D_{1}, x \in\left(D_{1}\right)_{k}$ for each $k$, and $\left(D_{1}\right)_{k} \subset \subset\left(D_{1}\right)_{k+1}$. For each $j$, denote $\left(D_{2}\right)_{k}^{j}=f_{j}\left(D_{1}\right)_{k}$. For a fixed $k$, we obtain by Theorem (a)(2)(3) the inequalities

$$
C_{\left(D_{1}\right)_{k}}^{n}(x, v) \geq C_{\left(D_{1}\right)_{k}^{j}}^{n}\left(x_{j}, d f_{j}(v)\right) \geq C_{\widetilde{D}}^{n}\left(x_{j}, d f_{j}(v)\right) .
$$

The last inequality on the above chain is valid for sufficiently large $j$. The reason is that when $j$ is sufficiently large, $f_{j}\left(\left(D_{1}\right)_{k}\right)=\left(D_{2}\right)_{k}^{j} \subset \widetilde{D}$ by Theorem (e), where $\widetilde{D}=V \cap D_{2}, p \in V$ is an open set in $\mathbb{C}^{n}$. It follows that for fixed $k$ and large $j$, we have the chain

$$
\frac{C_{\left(D_{1}\right)_{k}}^{n}(x, v)}{E_{D_{1}}^{n}(x, v)} \geq \frac{C_{\left(D_{2}\right)_{k}^{j}}^{n}\left(x_{j}, d f_{j}(v)\right)}{E_{D_{2}}^{n}\left(x_{j}, d f_{j}(v)\right)} \geq \frac{C_{\widetilde{D}}^{n}\left(x_{j}, d f_{j}(v)\right)}{E_{D_{2}}^{n}\left(x_{j}, d f_{j}(v)\right)}
$$

of inequalities (Theorem (a)(5) has been used here).

Observe that:

(i) By the volume decreasing property under holomorphic mappings (Theorem (a)(2)), we have $E_{\widetilde{D}}^{n}\left(x_{j}, \mathrm{df}_{j}(v)\right) \geq E_{D_{2}}^{n}\left(x_{j}, \mathrm{df}_{j}(v)\right)$ as the inclusion map $\widetilde{D} \hookrightarrow D_{2}$ is holomorphic. Therefore, we have

$$
\frac{C_{\left(D_{1}\right)_{k}}^{n}(x, v)}{E_{D_{1}}^{n}(x, v)} \geq \frac{C_{\widetilde{D}}^{n}\left(x_{j}, \mathrm{df}_{j}(v)\right)}{E_{\widetilde{D}}^{n}\left(x_{j}, \mathrm{df}_{j}(v)\right)} .
$$

(ii) Again by the strong pseudoconvexity of $p \in \partial D_{2}$, one obtains

$$
\frac{C_{\widetilde{D}}^{n}\left(x_{j}, \mathrm{df}_{j}(v)\right)}{E_{\widetilde{D}}^{n}\left(x_{j}, \mathrm{df}_{j}(v)\right)} \rightarrow 1 \quad \text { as } x_{j} \rightarrow p
$$

by Theorem (b).

(iii) If we let $k \rightarrow \infty$, then $C_{\left(D_{1}\right)_{k}}^{n}(x, v) \rightarrow C_{\left(D_{1}\right)}^{n}(x, v)$. This approximation property can be proved by elementary normal family argument.

(iv) It is always true that $C_{\left(D_{1}\right)}^{n}(x, v) / E_{\left(D_{1}\right)}^{n}(x, v) \leq 1$ by Theorem (a)(1).

Combining (i)-(iv), and letting $j \rightarrow \infty$ and then $k \rightarrow \infty$, one concludes that $1 \geq C_{\left(D_{1}\right)}^{n}(x, v) / E_{\left(D_{1}\right)}^{n}(x, v) \geq 1$, proving our claim.

\section{BIBLIOGRAPHY}

1. H. Alexander, Proper holomorphic mappings in $\mathrm{C}^{n}$, Indiana Univ. Math. J. 26 (1977), 137146.

2. E. Cartan, Groupes simples clos et ouverts et geometrie riemannienne, J. Math. Appl. 8 (1929), 1-33.

3. I. Graham, Boundary behavior of Carathéodory and Kobayashi metrics on s.p.c. domains in $\mathrm{C}^{n}$ with smooth boundary, Trans. Amer. Math. Soc. 207 (1975), 219-240. 
4. S. Kobayashi, Hyperbolic manifolds and holomorphic mappings, Marcel Dekker, 1970.

5. S. Pincuk, Proper holomorphic mappings of strictly pseudoconvex domains, Soviet Math. Dokl. 19 (1978), 804-807.

6. H. L. Royden, Remarks on Kobayashi metric, Lecture Notes in Math., Vol. 185, SpringerVerlag, New York, 1971, pp. 125-137.

7. B. Wong, Characterization of the unit ball in $\mathbb{C}^{n}$ by its automorphism group, Invent. Math. 41 (1977), 253-257.

8. __ Estimate of intrinsic measures on s.p.c. domains, unpublished manuscript.

Department of Mathematics, University of Toledo, Toledo, Ohio 43606

Department of MAThematics and Computer Science, University of CaliFORNIA, RIVERSIDE, CALIFORNIA 92521 